\title{
Targeted reversion of induced pluripotent stem cells from patients with human cleidocranial dysplasia improves bone regeneration in a rat calvarial bone defect model
}

\author{
Akiko Saito ${ }^{1 *}$, Akio Ooki ${ }^{2}$, Takashi Nakamura', Shoko Onodera ${ }^{1}$, Kamichika Hayashi ${ }^{3}$, Daigo Hasegawa ${ }^{3}$, \\ Takahito Okudaira ${ }^{3}$, Katsuhito Watanabe ${ }^{3}$, Hiroshi Kato ${ }^{3}$, Takeshi Onda ${ }^{3}$, Akira Watanabe ${ }^{3}$, Kenjiro Kosaki ${ }^{5}$, \\ Ken Nishimura ${ }^{6}$, Manami Ohtaka ${ }^{7}$, Mahito Nakanishi ${ }^{7}$, Teruo Sakamoto ${ }^{2}$, Akira Yamaguchi ${ }^{4}$, Kenji Sueishi ${ }^{2}$ \\ and Toshifumi Azuma ${ }^{1,4}$
}

\begin{abstract}
Background: Runt-related transcription factor 2 (RUNX2) haploinsufficiency causes cleidocranial dysplasia (CCD) which is characterized by supernumerary teeth, short stature, clavicular dysplasia, and osteoporosis. At present, as a therapeutic strategy for osteoporosis, mesenchymal stem cell (MSC) transplantation therapy is performed in addition to drug therapy. However, MSC-based therapy for osteoporosis in CCD patients is difficult due to a reduction in the ability of MSCs to differentiate into osteoblasts resulting from impaired RUNX2 function. Here, we investigated whether induced pluripotent stem cells (iPSCs) properly differentiate into osteoblasts after repairing the RUNX2 mutation in iPSCs derived from CCD patients to establish normal iPSCs, and whether engraftment of osteoblasts derived from properly reverted iPSCs results in better regeneration in immunodeficient rat calvarial bone defect models.

Methods: Two cases of CCD patient-derived induced pluripotent stem cells (CCD-iPSCs) were generated using retroviral vectors (OCT3/4, SOX2, KLF4, and c-MYC) or a Sendai virus SeVdp vector (KOSM302L). Reverted iPSCs were established using programmable nucleases, clustered regularly interspaced short palindromic repeats (CRISPR)/Casderived RNA-guided endonucleases, to correct mutations in CCD-iPSCs. The mRNA expressions of osteoblast-specific markers were analyzed using quantitative reverse-transcriptase polymerase chain reaction. iPSCs-derived osteoblasts were transplanted into rat calvarial bone defects, and bone regeneration was evaluated using microcomputed tomography analysis and histological analysis.
\end{abstract}

Results: Mutation analysis showed that both contained nonsense mutations: one at the very beginning of exon 1 and the other at the initial position of the nuclear matrix-targeting signal. The osteoblasts derived from CCD-iPSCs (CCD-OBs) expressed low levels of several osteoblast differentiation markers, and transplantation of these osteoblasts into calvarial bone defects created in rats with severe combined immunodeficiency showed poor regeneration. However, reverted iPSCs improved the abnormal osteoblast differentiation which resulted in much better engraftment into the rat calvarial bone defect.

(Continued on next page)

\footnotetext{
* Correspondence: ayokoyama@tdc.ac.jp

'Department of Biochemistry, Tokyo Dental College, Tokyo, Japan

Full list of author information is available at the end of the article
} 
(Continued from previous page)

Conclusions: Taken together, these results demonstrate that patient-specific iPSC technology can not only provide a useful disease model to elucidate the role of RUNX2 in osteoblastic differentiation but also raises the tantalizing prospect that reverted iPSCs might provide a practical medical treatment for CCD.

Keywords: Cleidocranial dysplasia, RUNX2, iPSCs, Osteoblasts, Osteogenesis, CRISPR/Cas,

\section{Background}

Cleidocranial dysplasia (CCD) is a dominantly inherited disorder characterized by patent fontanelles, wide cranial sutures, hypoplasia of the clavicles, short stature, and supernumerary teeth. In addition, other skeletal anomalies and osteoporosis are common [1, 2]. Runt-related transcription factor 2 (RUNX2), the gene responsible for CCD [3-5], encodes a transcription factor that is a member of the runt family of proteins [6]. These proteins contain a DNA-binding domain highly homologous to the Drosophila pair-rule gene runt [7]. In particular, RUNX2 is essential for the commitment of pluripotent mesenchymal cells toward osteoblasts (OBs), the bone-forming cells [8]. Some of the mutations found in CCD have been shown to interfere with the DNA-binding activity of RUNX2, whereas others have been found to alter the nuclear localization of the protein or to produce a mutant or truncated protein that is biologically inactive [9-11]. Runx2 gene expression must be tightly regulated to support skeletal health, although sufficient activity is necessary for adequate osteogenesis because reduced or excessive Runx2 activity may lead to osteoporosis [12]. Mice that are deficient in RUNX2 have complete absence of bone $[5,13,14]$, while those with haploinsufficiency of this transcription factor mimic some of the human CCD phenotypes, while others, such as supernumerary teeth, some skeletal abnormalities, and osteoporosis, have not been clearly demonstrated in mice [13].

Osteoporosis is a debilitating disease caused by systemic bone loss in the musculoskeletal system. Other than the anabolic parathyroid hormone drug, current Food and Drug Administration (FDA)-approved treatments are predominant agents that aim to inhibit bone resorption and prevent further bone loss. These types of treatment are associated with serious side effects and consequently there is a call for an alternative approach to treat osteoporosis [15]. The number of clinical trials involving mesenchymal stem cell (MSC)-based therapy has increased markedly over the last decade. This is because MSCs are multipotent adult stem cells that can be easily procured from various sources, such as bone marrow, adipose, and cord blood tissue [16].

However, in osteoporosis, the number of bone marrow MSCs that can differentiate into osteoblasts and form bone is significantly reduced. In addition, MSC derived from CCD patients seems to have a decreased ability to induce differentiation into osteoblasts due to haploinsufficiency of RUNX2.

Human induced pluripotent stem cells (hiPSCs) provide an iPSC-based novel disease model as well as a therapeutic option for the treatment of CCD [17]. We previously reported an efficient method to differentiate hiPSCs into osteogenic lineage cells [18] that supported the notion to generate CCD patient-derived iPSCs to elucidate the pathophysiology of CCD.

However, patient-derived iPSCs contain genetic mutations and exhibit poor osteogenic differentiation capability. Furthermore, hiPSCs have different genetic backgrounds and could exhibit heterogeneity, thereby limiting their use as model systems [19]. A technique has been developed to correct the defective gene in patient-derived iPSCs using programmable nucleases, i.e., clustered regularly interspaced short palindromic repeats (CRISPR)/Cas-derived RNA-guided endonucleases, which is now widely used for genomic editing of higher eukaryotic cells [20]. In this RNA-guided nuclease system, a user-defined single-guide RNA (sgRNA) directs the endonuclease Cas9 to a specific genomic target, where it cleaves chromosomal DNA. This cleavage activates endogenous cellular DNA repair pathways in a process known as homologous recombination, which gives rise to targeted correction of mutated genes [21]. In this report, we show the successful correction of the mutated RUNX2 gene, and show that this genetic correction gives rise to normal osteogenic potential in patient-derived iPSCs. Our evidence clearly reveals that reverted iPSCs could be used as a source for regeneration therapy.

Herein, we report the generation of two types of CCD patient-derived iPSCs, one with a heterozygous loss of DNA-binding activity of RUNX2 and another which supposedly alters nuclear localization of the RUNX2 protein. Both types showed impaired osteodifferentiation capabilities. We further generated reverted iPSCs to restore the proper osteodifferentiation potential not only in vitro but also in vivo. These iPSCs should prove valuable for elucidating disease mechanisms and providing an iPSC-based novel therapeutic option for the treatment of CCD or other diseases related to the Runx2 gene. 


\section{Methods}

\section{Cell culture}

All experimental procedures described in this manuscript were approved by the Ethics Committee of Tokyo Dental College (permit no. 533). Primary human oral fibroblasts (OFs) were prepared from oral mucosal tissue $(5 \times 10 \mathrm{~mm})$ resected from CCD patients, which were then cultured and subjected to reprogramming by infecting with retroviral vectors (OCT3/4, SOX2, KLF4, and c-MYC) or a Sendai virus SeVdp vector (KOSM302L) as described previously [22-24]. All iPSCs were maintained in human embryonic stem cell (ESC) media (Dulbecco's modified Eagle's medium/nutrient mixture F-12 + GlutaMAX media supplemented with $20 \%$ knockout serum replacement media, $1 \%$ L-glutamine, $1 \%$ nonessential amino acids, $1 \%$ penicillin-streptomycin, $0.1 \mathrm{mM}$ 2-mercaptoethanol, and $5 \mathrm{ng} / \mathrm{ml}$ fibroblast growth factor (FGF)-2).

The efficient method of differentiating hiPSCs into osteogenic lineage cells is shown in our previous study [18] as well as in Fig. 2a. For osteoblast differentiation, cells were cultured in osteoblast differentiation medium (OBM), which consisted of alpha-MEM supplemented with $10 \%$ fetal bovine serum (FBS), $50 \mathrm{mg} / \mathrm{ml} \mathrm{L}$-ascorbic acid, $10 \mathrm{mM} \beta$-glycerophosphate, and $10 \mathrm{nM}$ dexamethasone, supplemented with cytokines $(25 \mathrm{ng} / \mathrm{ml}$ FGF-2, $1 \mathrm{ng} / \mathrm{ml}$ transforming growth factor (TGF)- $\beta 1$, $100 \mathrm{ng} / \mathrm{ml}$ insulin-like growth factor (IGF)-1) for 12 days. OBM containing fresh cytokines was resupplied every 3 days.

Primary human osteoblasts (HOBs) derived from normal bone tissue were purchased from Takara Bio Inc. (Shiga, Japan) and cultured in osteoblast growth medium (Takara Bio Inc.).

\section{Mutation analysis}

Exons $1-7$ of the RUNX2 gene were amplified by polymerase chain reaction (PCR) under standard conditions for analyzing CCD case 1 (CCD1) and CCD case 2 (CCD2). The primers used for genomic PCR amplification and sequencing are described elsewhere [25].

\section{CRISPR/sgRNA design and construction}

We explored the sgRNA using the CRISPRdirect bioinformatics tool against the human RUNX2 locus located on chromosome 6 . Complete sequences of the target sites of the sgRNA are provided in Fig. 1g. The targeting donor vector was constructed based on a DT-A/conditional knockout FW plasmid (RIKEN Center for Developmental Biology, Kobe, Japan) with several modifications. The PCR-amplified fragments from genomic DNA of a healthy individual were inserted into the restriction enzyme-digested plasmid.
CRISPR/sgRNA transfections for reversion in iPSCs

Transfection of the CRISPR/sgRNA plasmid into iPSCs was performed as described previously [26]. Electroporation was performed using a Neon Transfection System (Thermo Fisher Scientific, Waltham, MA, USA) according to the manufacturer's instructions. Briefly, iPSCs were harvested by treating with $0.05 \%$ trypsin-ethylenediaminetetraacetic acid (EDTA) solution (Thermo Fisher Scientific). Cells $\left(1 \times 10^{6}\right)$ were suspended in $100 \mu \mathrm{l}$ electroporation buffer containing $5 \mu \mathrm{g}$ of the donor plasmid and $5 \mu \mathrm{g}$ of the CRISPR/sgRNA expression plasmid, and electroporated (condition: 1050 $\mathrm{V}, 20 \mathrm{~ms}$, two pulses). After electroporation, the cells were subsequently plated on an iMatrix-511 (Nippi Inc., Tokyo, Japan)-coated 100-mm dish and cultured in StemFit ${ }^{\oplus}$ AK01 medium (Ajinomoto, Tokyo, Japan) supplemented with ROCK inhibitor (Y-27632; Wako Pure Chemical Industries Ltd.) for the first $24 \mathrm{~h}$. Geneticin selection $(300 \mu \mathrm{g} / \mathrm{ml})$ was started $72 \mathrm{~h}$ after electroporation. Individual colonies were picked and expanded for 10 days after electroporation.

\section{RNA isolation, reverse transcriptase PCR, and quantitative PCR}

Total RNA was extracted using QIAzol reagent (Qiagen, Hilden, Germany) according to the manufacturer's instructions. Complementary DNA (cDNA) was synthesized using a high-capacity cDNA reverse transcription kit (Thermo Fisher Scientific). To confirm the expression of markers of ESCs and three germ layers, PCR was performed with Ex-Taq polymerase (Takara Bio, Inc.). $\beta$-actin was used as an internal control. All primer sets are described elsewhere [24]. Quantitative reverse transcriptase (qRT)-PCR was performed using Premix Ex-Taq reagent (Takara Bio, Inc.) according to the manufacturer's instructions. 18S rRNA was used as an internal control. All primer sequences are shown in Table 1. The relative expression of genes of interest was estimated using the $\Delta \Delta \mathrm{Ct}$ method.

\section{Immunofluorescent staining}

Cells cultured on cover glasses were fixed in $4 \%$ paraformaldehyde in phosphate-buffered saline (PBS) for 15 min, permeabilized with ice-cold 100\% methanol for 10 min at $-20{ }^{\circ} \mathrm{C}$, and then blocked with $5 \%$ normal goat serum/0.3\% Triton X-100 in PBS for $1 \mathrm{~h}$. Subsequently, the cells were incubated with anti-RUNX2 antibody (dilution 1:1600; Cell Signaling Technology, Inc., Beverly, MA, USA), followed by Alexa Fluor ${ }^{\circ} 488$-conjugated goat anti-rabbit or mouse immunoglobulin G. Localization of the RUNX2 protein was visualized by immunofluorescent microscopy (UPM AxiophoT2; Carl Zeiss Microscopy, LLC, Thornwood, NY, USA). 
a

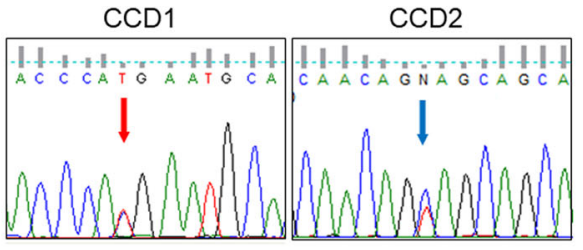

R391X

Q67X
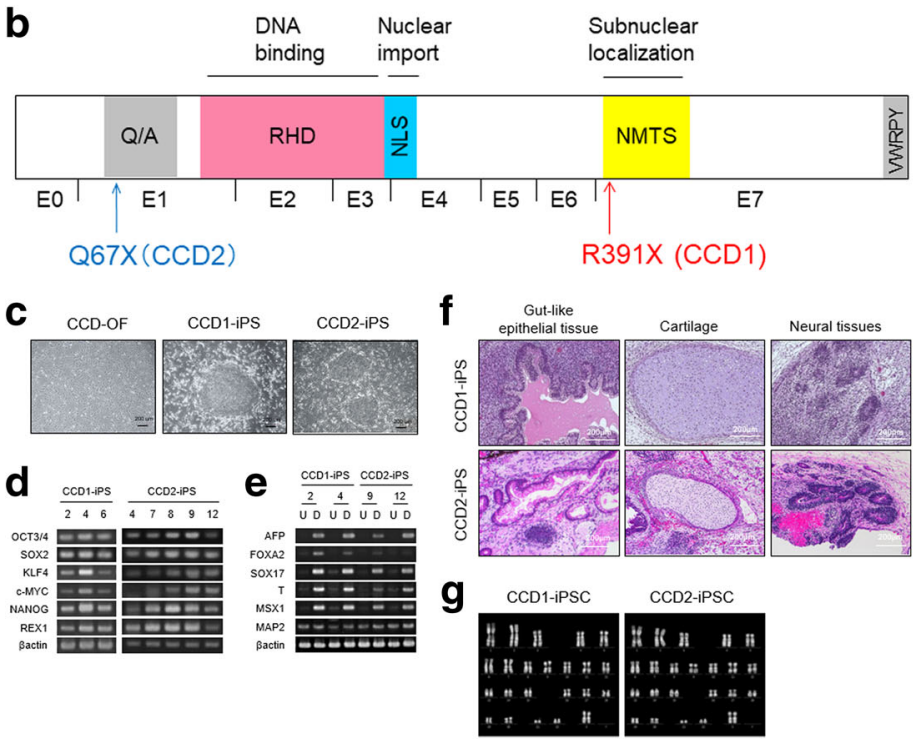

h

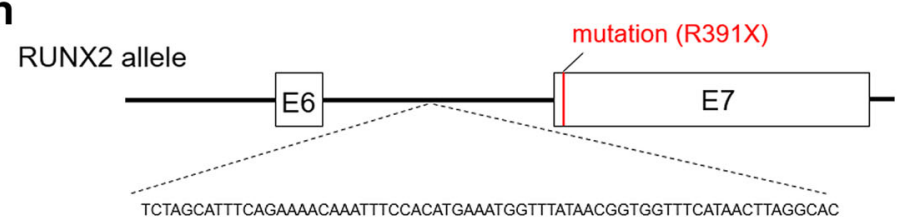

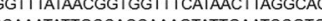

CRISPR/sgRNA CATGAAATGGTTTATAACGGTGG
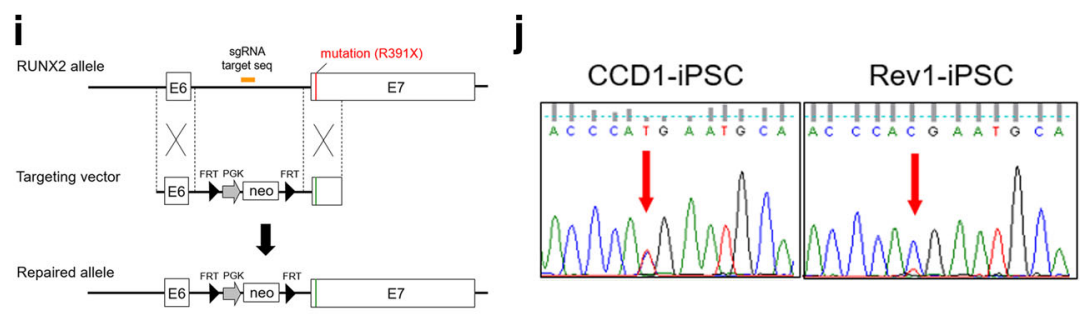

k
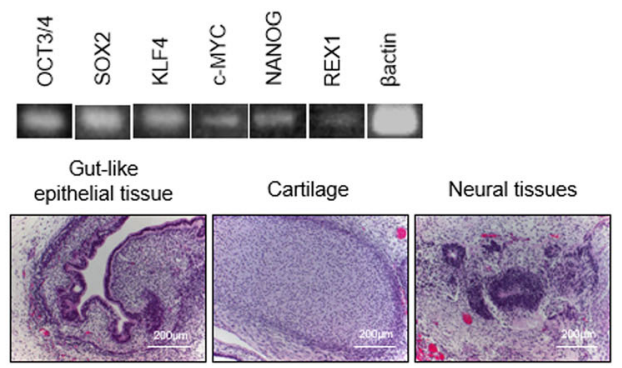

Rev1-iPSC

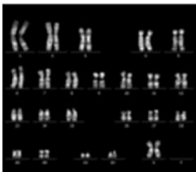

Fig. 1 (See legend on next page.) 
(See figure on previous page.)

Fig. 1 Generation of cleidocranial dysplasia (CCD) patient-specific iPSCs and genome editing. a ldentification of heterozygous runt-related transcription factor 2 (RUNX2) mutations in each CCD. b Functional domains of RUNX2 proteins and RUNX2 exon organization. c Morphology of established CCD patient-derived induced pluripotent stem cells (CCD-iPS). $\mathbf{d}$-f Confirmation of the pluripotency of the CCD-iPSCS. $\mathbf{d}$ RT-PCR analysis of ESC marker genes. e RT-PCR analyses of various differentiation markers for the three germ layers. Target genes included AFP, FOXA2, and SOX17 (endoderm), T and MSX1 (mesoderm), and MAPs (ectoderm). $\beta$-actin was used as an internal control. f Teratoma formation and histology. Teratoma contained tissues of three embryonic germ layers: cartilage (mesoderm), gut-like epithelium tissues (endoderm), and neural tube-like structures (ectoderm). $\mathbf{g}$ Karyotype analysis (Q-band) of CCD1- and CCD2-iPSCs. h Clustered regularly interspaced short palindromic repeats (CRISPR)/single-guide RNA (sgRNA) targeting of the RUNX2 gene on chromosome 6. i CRISPR-mediated genome editing of CCD1-iPSCs. $\mathbf{j}$ Confirmation of the RUNX2 gene correction by sequence analysis. $\mathbf{k}$ Confirmation of the pluripotency of the Reverted iPSCs (Rev1-iPSCs). I Karyotype analysis (Q-band) of Rev1-iPSCs. Abbreviations: D, differentiated; $\mathrm{U}$, undifferentiated; $\mathrm{Q} / \mathrm{A}$, glutamine/alanine-rich domain; RHD, runt homology domain; NLS, nuclear localization signal; NMTS, nuclear matrix-targeting signal; FRT, flippase recognition target; PGK, phosphoglycerate kinase

\section{Preparation of scaffold/iPSC-derived OB complex for transplantation}

Osteogenic differentiation from iPSCs was performed according to Fig. 3a. After 4 weeks, the iPSC-derived OBs were dissociated with $0.5 \mathrm{mg} / \mathrm{ml}$ collagenase type IV for $30 \mathrm{~min}$ and $0.25 \%$ trypsin-EDTA for $5 \mathrm{~min}$ at $37^{\circ}$ C. To facilitate the self-assembly of a peptide nanofiber scaffold-derived three-dimensional (3D) culture, we used PuraMatrix (BD Biosciences, Cambridge, MA, USA) to encapsulate the cells; $10 \mu \mathrm{l}$ of freshly dissociated cells (2 $\times 10^{5}$ ) suspended in serum-free media was mixed with $10 \mu \mathrm{l}$ of the $2.5 \%$ PuraMatrix. Gelation was initiated after the peptide solution was mixed with the cell suspension, thereby resulting in cell encapsulation inside the nanofiber hydrogel.

\section{Transplantation of scaffold/iPSC-derived OB complex}

All procedures performed with live animals conformed to the ethical guidelines established by the Japanese Council on Animal Care and were approved by the Animal Care Committee of Tokyo Dental College (permit no. 290401). Seven-week-old male F344/NJcl$\mathrm{rnu} / \mathrm{rnu}$ rats were obtained from Clea Japan, Inc. (Tokyo, Japan). After anesthesia induction with 4\% sevoflurane (Maruishi Pharmaceutical Co. Ltd., Osaka, Japan) inhalation, the rats were further anesthetized by intraperitoneal injection with sodium pentobarbital $(30 \mathrm{mg} / \mathrm{kg}$ body weight; somnopentyl; Kyoritsu Seiyaku, Tokyo, Japan). Rat calvarial bone defects were created as previously described [27]. Scaffold/iPSC-derived OB complex was injected into the bone defect. The rats receiving transplantation were sacrificed at 4 weeks and subjected to radiographical and histological analyses.

\section{Radiographical analyses and histological assessment}

Microcomputed tomography (CT) parameters were as follows: X-ray source, $90 \mathrm{kV} / 100 \mu \mathrm{A}$; rotation, $360^{\circ}$; exposure time, $17 \mathrm{~s}$; and voxel size, $50 \times 50 \times 50 \mu \mathrm{m}$ (R- $\mu \mathrm{CT}^{\oplus}$; Rigaku Corporation, Tokyo, Japan). CT images were compiled and three-dimensional images were rendered using the TRI/3D-BON system (Ratoc System Engineering Co. Ltd., Tokyo, Japan) as described previously [27]. Coronal sections (thickness $=5 \mu \mathrm{m}$ ) through the center of each circular defect were prepared and Villanueva-Goldner (V-G) staining [28] was performed.

\section{Statistical analysis}

All data are expressed as the mean \pm standard deviation (SD). When analysis of variance indicated differences among the groups, multiple comparisons among the experimental groups were performed using

Table 1 Primers used for quantitative reverse transcriptase polymerase chain reaction

\begin{tabular}{llll}
\hline Gene symbol & GenBank accession no. & Forward primer sequence & Reverse primer sequence \\
\hline RUNX2 & NM_001024630.2 & gtgcctaggcgcatttca & gctcttcttactgagagtggaagg \\
ALP & NM_000478.3 & caacctggggaggagac & gcattggtgttgtacgtcttg \\
COL1A1 & NM_000088.3 & gggattccctggacctaaag & gggattccctggacctaaag \\
OSX & NM_152860.1 & catctgcctggctccttg & caggggactggagccata \\
OC & NM_199173.3 & tgagagccctcacactcctc & acctttgctggactctgcac \\
MSX2 & NM_002449.4 & tcggaaaattcagaagatgga & caggtggtagggctcatatgtc \\
DLX5 & NM_005221.5 & ctacaaccgcgtcccaag & gccattcaccattctcacct \\
DLX3 & NM_005220.2 & gagcctcctaccggcaatac & tcctccttcaccgacactg \\
TWIST1 & NM_000474.3 & agctacgccttctcggtct & ccttctctggaaacaatgacatc \\
18SrRNA & M11188.1 & cggacaggattgacagattg & cgctccaccaactaagaacg \\
\hline
\end{tabular}


the Bonferroni test. Statistical significance was defined as $p<0.05$.

\section{Results}

Sequencing analysis of the genomic DNA extracted from the CCD-OFs revealed a heterozygous nonsense mutation (R391X as CCD1, Q67X as CCD2) in the RUNX2 gene (Fig. 1a). This mutation corresponds with the functional domain of the RUNX2 protein, nuclear matrix-targeting signal (NMTS) (CCD1), and the glutamine/alanine-rich domain (Q/A) (CCD2) (Fig. 1b). More than three iPSC lines were established and analyzed for each $\mathrm{CCD}$ patient. Patient-specific iPSCs from CCD-OFs (Fig. 1c) were generated using retroviral or Sendai viral vectors that encode the four Yamanaka factors [23, 24]. All the retroviral or Sendai viral iPSC lines suppressed transgenes (data not shown). The pluripotency of the CCD-iPSC lines was confirmed as shown in Fig. 1d-f. CCD-iPSCs displayed basic properties of pluripotent cells, as indicated by the expression of the embryonic stem cell markers OCT3/4, $N A N O G$, and REX1 (Fig. 1d), the expression of the three germ-layer differentiation markers in embryoid bodies (Fig. 1e), the ability to form teratomas (Fig. 1f), and a normal karyotype (Fig. 1g). Reverted iPSC (Rev1-iPSC) lines were generated using CRISPR/Cas9-derived RNA-guided endonucleases (Fig. 1h, i). RUNX2 gene correction was confirmed by sequence analysis (Fig. 1j). Rev1-iPSCs were maintained in a pluripotent state, as indicated by the expression of the pluripotency markers, and displayed the ability to form teratomas (Fig. 1k), and a normal karyotype (Fig. 11).

Osteogenic differentiation from iPSCs was performed according to our previous report [18] and Fig. 2a. CCD-OBs showed a decrease in the nuclear localization of RUNX2 (Fig. 2b). However, RUNX2 localization was restored in Rev1-OBs to the same level as normal primary HOBs (Fig. 2b). Furthermore, we examined Runx2 localization in calvarial OBs derived from Runx2 wildtype (Runx2 $2^{+/+}$) and heterozygous knock-out (Runx2 $2^{+/-}$) mice. As a result, Runx2 localization was decreased in Runx $2^{+/-}$-OBs compared to Runx $2^{+/+}$-OBs (Additional file 1: Figure S1). We determined the expression of OB-specific markers to confirm OB differentiation. As expected, in OBM with FGF-2, TGF- $\beta 1$, and IGF-1 greatly increased the alkaline phosphatase (ALP) activity of Rev1-iPSCs, but not CCD1-iPSC, in a time-dependent manner (Fig. 2c). Furthermore, we examined ALP activity of primary $\mathrm{HOBs}$ and mouse $\mathrm{OBs}$ (mOBs) as controls. As a result, the ALP activity of Rev1-iPSCs cultured in OBM for 12 days was at the same level as HOBs and Runx $2^{+/+}$-mOBs. On the other hand, the ALP activity of Runx $2^{+/-}$-mOBs was decreased similarly to CCD1-iPSCs (Additional file 2: Figure S2). As compared with that in CCD1-iPSCs, the expression levels of the
OB-specific markers $A L P$, OSX, and $O C$ were markedly upregulated only in Rev1-iPSCs cultured in OBM for 9 days (Fig. 2d). The mRNA expression level of RUNX2 was higher in CCD1-iPSCs than in Rev1-iPSCs (Fig. 2e). We next investigated major homeodomain (HD) proteins in osteogenesis, such as $D L X 5, D L X 3, M S X 2$, and TWIST1 [29]. The expression levels of DLX5, MSX2, and TWIST1 mRNA molecules were sharply increased in Rev1-iPSCs cultured in OBM after 9 days, but not in CCD1-iPSCs (Fig. 2e). The expression level of DLX3 was higher in CCD1-iPSCs than in Rev1-iPSCs (Fig. 2e). These observations indicated that the osteolineage differentiation of CCD1-iPSCs was significantly delayed, relative to that of Rev1-iPSCs.

MicroCT images of the calvaria at 4 weeks after transplantation are shown in Fig. 3b. Re-ossification developed via growth extension from the bony rims at the lateral edges of the bone defects. Newly generated bone was observed 4 weeks after surgery in the Rev group. Minimal new bone was observed in the two CCD groups (Fig. 3b). The bone volume (BV) and bone mineral content (BMC) were significantly higher in the Rev group than in the CCD groups (Fig. 3c). On the other hand, there was no difference in bone mineral density (BMD) (Fig. 3c). Histological examination by V-G staining provides uniform and reproducible results with mineralized or undecalcified bone. Mineralized bone tissues are stained green and nonmineralized osteoid tissues are stained red by V-G stain (Fig. 3d). Newly formed bone was observed around the margin and along the intracranial periosteum, as well as in the center of the bone defects, including around the top portion of the defect area in the Rev group. In the CCD groups, there was comparatively less newly formed bone (Fig. 3d), indicating that CCD-iPSCs may have delayed osteodifferentiation.

\section{Discussion}

In this study we present two major results. First, we successfully corrected the mutated Runx 2 gene in iPSCs using the CRISPR/Cas method. Second, the reverted iPSCs that we generated exhibited better osteogenic properties both in vivo and in vitro, suggesting that these iPSCs provide not only an ideal source for studying CCD but also provide an iPSC-based novel therapeutic option for the treatment of CCD or other diseases related to the RUNX2 gene.

We showed that CCD-iPSCs were found to have poor osteogenic differentiation capability, as the expression levels of transcription factors important for osteodifferentiation (i.e., HD protein, OSX, TWIST1, MSX2, and $D L X 5)$ were not increased in the proper manner observed in Rev-iPSCs cultured in OBM after 9 days. An in vivo experiment (calvarial defects model) revealed the 

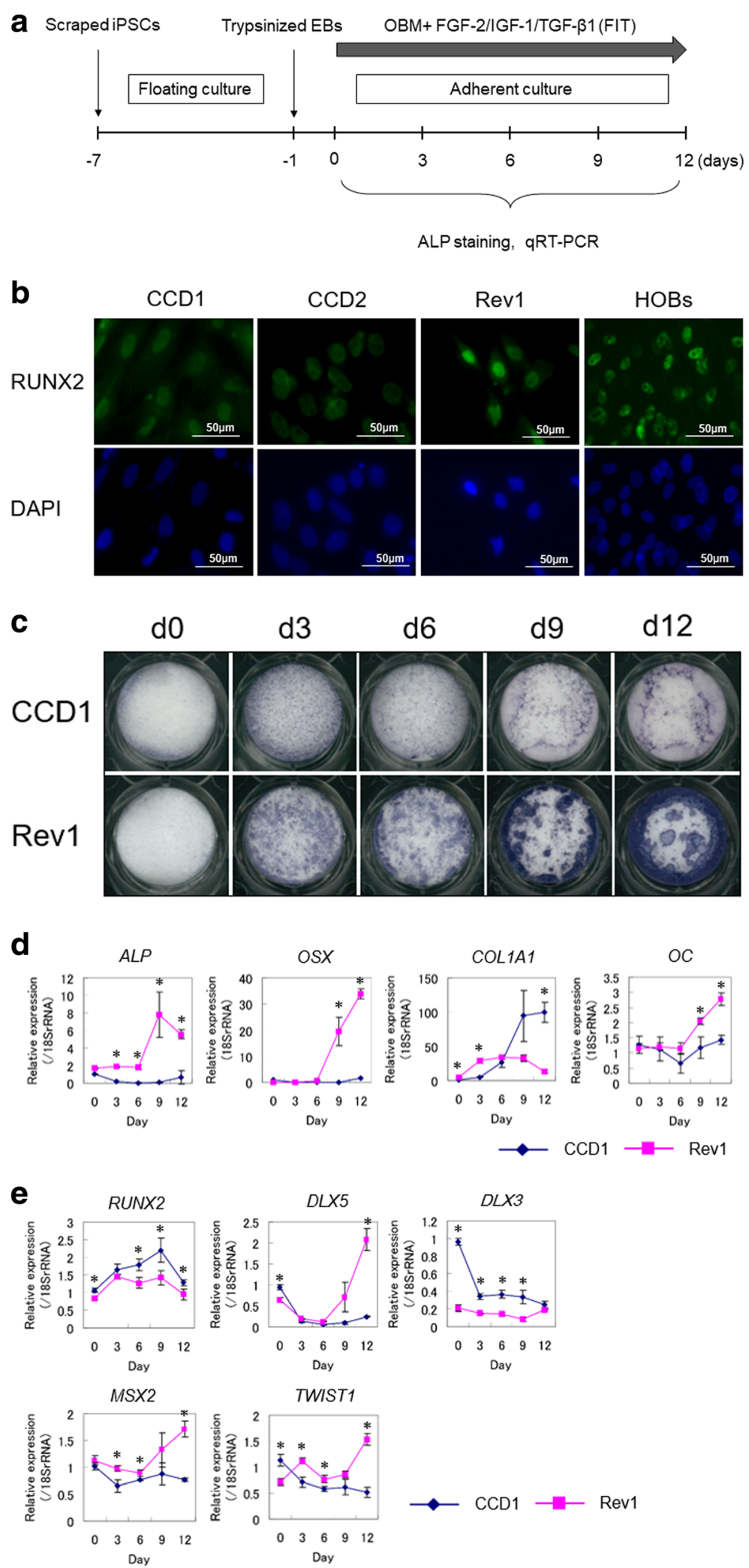

Fig. 2 (See legend on next page.) 
(See figure on previous page.)

Fig. 2 Osteoblastic differentiation of induced pluripotent stem cells (iPSCs) in vitro. a Schedule of osteogenic differentiation. b Runt-related transcription factor 2 (RUNX2) localization by immunofluorescent microscopy. Single cells from embryoid bodies (EBs) were cultured with osteoblast differentiation medium (OBM) containing cytokines for 12 days and stained for RUNX2 (green) and the nuclei (blue). c Staining of alkaline phosphatase (ALP) activity (days (d)0, 3, 6, 9, and 12). $\mathbf{d}$ qRT-PCR analysis of RUNX2 target genes. e qRT-PCR analysis of RUNX2 and transcription factors. Values are presented as the mean $\pm \mathrm{SD}(n=3) .{ }^{*} p<0.05$. FGF fibroblast growth factor, HOB human osteoblast, IGF insulin-like growth factor, TGF transforming growth factor

poor regeneration capability of CCD-OBs relative to that of Rev-OBs.

In the present study, two types of CCD-iPSC derived from patients enrolled in this study were generated and each had a stop codon insertion mutation in the Q/Arich domain or NMTS domain. Nonsense mutation in the Q/A-rich domain resulted in complete loss of runt domain, which is a responsive sequence for binding to Runx2 target cic-element. On the other hand, NMTS is an important domain for nuclear localization [30]. Deficiency of this domain significantly impeded nuclear localization of RUNX2 and reduced the coordinated action of the Smad signaling [30, 31]. These Runx2 functions were some of the most important functional domains in the Runx2 gene. The deficiency of any domain similar to a loss of function of the transcription factor of RUNX2 has been reported to result in failure of bone and cartilage differentiation [30-33]. Similarly, we found that the osteodifferentiation capability of the two cases of CCD-iPSCs we examined were poor.

We showed that Rev-iPSCs, which could have restored the proper osteodifferentiation potential, were generated by the CRISPR/Cas method. We sequenced and confirmed the corrected mutated sequence. These Rev-iPSCs still maintained pluripotencies but gained better osteogenic abilities. iPSCs provide an attractive platform for the study of rare diseases, including CCD. Patient-derived iPSCs have great potential as a disease model because they
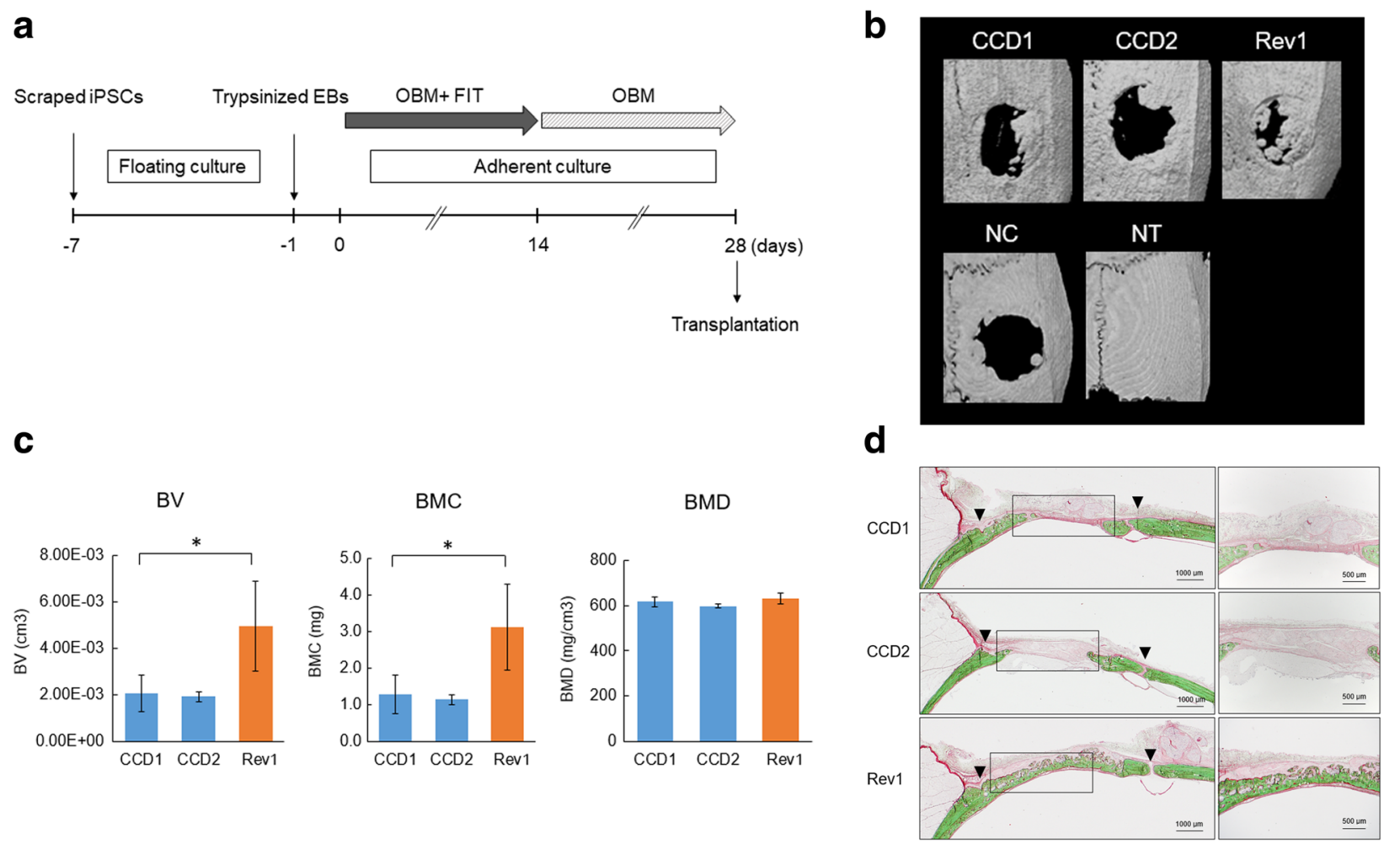

Fig. 3 Transplantation of induced pluripotent stem cell (iPSC)-induced osteoblasts (OBs) and bone regeneration. a Transplantation protocol for rat calvarial bone defects. b MicroCT images of the calvaria at 4 weeks after transplantation in the CCD1, CCD2, and Rev1 groups $(n=3)$. c MicroCT analysis of bone regeneration. Comparison of new bone volume $\left(\mathrm{cm}^{3}\right)$ in the regions of interest of three groups. Values are presented as the mean \pm SD $(n=3)$. ${ }^{*} p<0.05$. d V-G staining images at 4 weeks after transplantation. Top: CCD1 group. Middle: CCD2 group. Lower: Rev1 group. Magnified images of the new bone area are shown on the right. BMC bone mineral content, BMD bone mineral density, BV bone volume, EB embryoid body, NC nontransplanted defect (negative control), FIT fibroblast growth factor/insulin-like growth factor/transforming growth factor, NT nontreated control, OBM osteoblast differentiation medium 
can generate unlimited quantities of cells. Furthermore, genetic manipulations, such as the introduction of exogenous genes and specific correction of a defined mutation in established iPSCs, could lead to novel cellular therapies. To date, there have been few reports of successfully generated corrected iPSCs (Rev-iPSCs) from patients [34-37]. Another important reason to generate Rev-iPSCs is their utility as a control for patient-derived iPSCs in research. Therefore, we established Rev-iPSCs as control cells from these CCD-iPSCs using the CRISPR/Cas tool.

RUNX2-HD protein complexes regulate target genes, and RUNX2 has been characterized as a major hub of HD protein regulation [29, 38-41]. Hojo et al. also reported that osterix (Osx) is restricted to bone-forming vertebrates, where it acts as a Dlx co-factor in OB specification [39]. We investigated major HD proteins in osteogenesis from human-derived iPSCs and found that insufficiency of RUNX2 might disrupt the early osteogenic circuitry for commitment of the bone cell phenotype in the differentiation from iPSC to osteolineage cells. Recent reports also described that aged mice required full Runx2 gene dosage for cancellous bone regeneration after bone marrow ablation [42]. Experiments with a calvarial bone defect model using severe combined immunodeficiency rats to transplant CCDOBs showed poor bone formation, while reverted CCD-OBs showed substantially improved bone regeneration. However, there was no significant difference in BMD among all three groups. Thus, RUNX2 may have a weak effect on BMD owing to its regulation, which covers a relatively early stage, but not late stage, of osteoblast differentiation. These findings suggest that the haploinsufficiency of RUNX2 has a significant effect on the early stage of membrane ossification, which causes clinical symptoms in patients with $C C D$, and that reverted iPSCs provide an iPSC-based novel therapeutic option for the treatment of CCD.

\section{Conclusions}

iPSCs were generated from patients with CCD, and Rev-iPSCs with corrected mutations were created by CRISPR/Cas-mediated genome editing. These iPSCs can not only provide a useful disease model to elucidate the role of RUNX2 in osteoblastic differentiation but also raise the tantalizing prospect that reverted iPSCs might provide a practical medical treatment for CCD.

\section{Additional files}

Additional file 1: Figure S1. RUNX2 localization in primary calvarial osteoblasts (OBs) derived from Runx 2 wild-type mice (Run $\times 2^{+/+} \mathrm{mOBs}$ ) and Runx2 heterozygous knock-out mice (Run $\times 2^{+/-} \mathrm{mOBs}$ ) by immunofluorescent microscopy. RUNX2 (green) and the nuclei (blue). (TIFF 237 kb)
Additional file 2: Figure S2. Staining of ALP activity in $\mathrm{HOBS}$ and mOBs (Run $\times 2^{+/+}$and Runx $2^{+/-}$). (TIFF $350 \mathrm{~kb}$ )

\section{Abbreviations}

ALP: Alkaline phosphatase; BMC: Bone mineral content; BMD: Bone mineral density; BV: Bone volume; CCD: Cleidocranial dysplasia;

CDNA: Complementary DNA; CRISPR: clustered regularly interspaced short palindromic repeats; CT: Computed tomography;

EDTA: Ethylenediaminetetraacetic acid; ESC: Embryonic stem cell; FGF: Fibroblast growth factor; hiPSC: Human induced pluripotent stem cell; HOB: Human osteoblast; IGF: Insulin-like growth factor; iPSC: Induced pluripotent stem cell; mOB: Mouse osteoblast; MSC: Mesenchymal stem cell; NMTS: Nuclear matrix-targeting signal; OB: Osteoblast; OBM: Osteoblast differentiation medium; OF: Oral fibroblast; OSX: Osterix; PBS: Phosphatebuffered saline; PCR: Polymerase chain reaction; qRT-PCR: Quantitative reverse transcriptase polymerase chain reaction; RUNX2: Runt-related transcription factor 2; SD: Standard deviation; sgRNA: Single-guide RNA; TGF: Transforming growth factor; V-G: Villanueva-Goldner

\section{Acknowledgments}

The authors would like to thank Ajinomoto Co. Ltd. for technical advice. The authors would like to thank Enago (http://www.enago.jp/) for the English language review.

\section{Funding}

This work was supported by JSPS KAKENHI (C) (grant no. 25462900) and by JSPS KAKENHI for young scientists (B) (grant nos. 26861686, 26861560, 16 K20427, and 16 K20428).

\section{Availability of data and materials}

The datasets supporting the results of this article are included within the article and Additional files.

\section{Authors' contributions}

AS and TA conceived and designed the study. Field work was carried out by $\mathrm{AS}$ and $\mathrm{AO}$. All analyses were completed by AS with support from $\mathrm{KH}$ and HK (transplantation), DH (teratoma formation), TO and KW ( $\mu$ CT analysis), TO, AW, and TS (generation of patient fibroblasts), TN (gene editing), SO and KK (mutation analysis), and $\mathrm{OM}, \mathrm{KN}$, and MN (generation of Sendai virus vector). $\mathrm{AY}$ and $\mathrm{KS}$ supervised the project. All authors contributed to the discussion of the results. The manuscript was written by AS with major input from TA. All authors read and approved the final manuscript.

\section{Ethics approval and consent to participate}

Human samples were obtained and processed after informed consent was provided in accordance with the Declaration of Helsinki and under the approval of the Ethics Committee for clinical research at the Tokyo Dental College (Tokyo Japan) (permit no. 533). All procedures performed with live animals conformed to the ethical guidelines established by the Japanese Council on Animal Care and were approved by the Animal Care Committee of Tokyo Dental College (permit no. 290401).

Consent for publication

Informed consent was obtained from all patients.

\section{Competing interests}

The authors declare that they have no competing interests.

\section{Publisher's Note}

Springer Nature remains neutral with regard to jurisdictional claims in published maps and institutional affiliations.

\section{Author details}

${ }^{1}$ Department of Biochemistry, Tokyo Dental College, Tokyo, Japan. ²Department of Orthodontics, Tokyo Dental College, Tokyo, Japan. ${ }^{3}$ Department of Oral and Maxillofacial Surgery, Tokyo Dental College, Tokyo, Japan. ${ }^{4}$ Oral Health Science Center, Tokyo Dental College, Tokyo, Japan. ${ }^{5}$ Center for Medical Genetics, Keio University School of Medicine, Tokyo, Japan. ${ }^{6}$ Laboratory of Gene Regulation, Faculty of Medicine, University of Tsukuba, Ibaraki, Japan. ${ }^{7}$ Biotechnology Research Institute for Drug Discovery, 
National Institute of Advanced Industrial Science and Technology (AIST), Tsukuba, Ibaraki, Japan.

\section{Received: 27 September 2017 Revised: 24 November 2017} Accepted: 19 December 2017 Published online: 22 January 2018

\section{References}

1. Mundlos S. Cleidocranial dysplasia: clinical and molecular genetics. J Med Genet. 1999:36:177-82

2. Mendoza-Londono R, Lee B. Cleidocranial dysplasia. GeneReviews ${ }^{\oplus}$. Seattle: University of Washington; 1993.

3. Lee B, Thirunavukkarasu K, Zhou L, Pastore L, Baldini A, Hecht J, et al. Missense mutations abolishing DNA binding of the osteoblast-specific transcription factor OSF2/CBFA1 in cleidocranial dysplasia. Nat Genet. 1997; 16:307-10.

4. Mundlos S, Otto F, Mundlos C, Mulliken JB, Aylsworth AS, Albright S, et al. Mutations involving the transcription factor CBFA1 cause cleidocranial dysplasia. Cell. 1997;89:773-9.

5. Komori T, Yagi H, Nomura S, Yamaguchi A, Sasaki K, Deguchi K, et al. Targeted disruption of Cbfa1 results in a complete lack of bone formation owing to maturational arrest of osteoblasts. Cell. 1997;89:755-64.

6. Levanon D, Negreanu V, Bernstein Y, Bar-Am I, Avivi L, Groner Y. AML1, $A M L 2$, and AML3, the human members of the runt domain gene-family: cDNA structure, expression, and chromosomal localization. Genomics. 1994; 23:425-32.

7. Nüsslein-Volhard C, Wieschaus E. Mutations affecting segment number and polarity in Drosophila. Nature. 1980;287:795-801.

8. Komori T. Runx2, a multifunctional transcription factor in skeletal development. J Cell Biochem. 2002;87:1-8.

9. Vaughan T, Pasco JA, Kotowicz MA, Nicholson GC, Morrison NA. Alleles of RUNX2/CBFA1 gene are associated with differences in bone mineral density and risk of fracture. J Bone Miner Res. 2002;17:1527-34.

10. Vaughan T, Reid DM, Morrison NA, Ralston SH. RUNX2 alleles associated with BMD in Scottish women; interaction of RUNX2 alleles with menopausal status and body mass index. Bone. 2004;34:1029-36.

11. Doecke JD, Day CJ, Stephens ASJ, Carter SL, van Daal A, Kotowicz MA, et al. Association of functionally different RUNX2 P2 promoter alleles with BMD. J Bone Miner Res. 2006;21:265-73.

12. Liu W, Toyosawa S, Furuichi T, Kanatani N, Yoshida C, Liu Y, et al. Overexpression of Cbfa1 in osteoblasts inhibits osteoblast maturation and causes osteopenia with multiple fractures. J Cell Biol. 2001;155:157-66.

13. Otto F, Thornell AP, Crompton T, Denzel A, Gilmour KC, Rosewell IR, et al. Cbfa1, a candidate gene for cleidocranial dysplasia syndrome, is essential for osteoblast differentiation and bone development. Cell. 1997;89:765-71.

14. Glotzer DJ, Zelzer E, Olsen BR. Impaired skin and hair follicle development in Runx2 deficient mice. Dev Biol. 2008;315:459-73.

15. Antebi B, Pelled G, Gazit D. Stem cell therapy for osteoporosis. Curr Osteoporos Rep. 2014;12:41-7.

16. Trounson A, Thakar RG, Lomax G, Gibbons D. Clinical trials for stem cell therapies. BMC Med. 2011;9:52.

17. Yamasaki S, Hamada A, Akagi E, Nakatao H, Ohtaka M, Nishimura K, et al. Generation of cleidocranial dysplasia-specific human induced pluripotent stem cells in completely serum-, feeder-, and integration-free culture. Vitr Cell Dev Biol Anim. 2016;52:252-64.

18. Ochiai-Shino H, Kato H, Sawada T, Onodera S, Saito A, Takato T, et al. A novel strategy for enrichment and isolation of osteoprogenitor cells from induced pluripotent stem cells based on surface marker combination. PLoS One. 2014;9:e99534.

19. Rouhani F, Kumasaka N, de Brito MC, Bradley A, Vallier L, Gaffney D. Genetic background drives transcriptional variation in human induced pluripotent stem cells. PLoS Genet. 2014;10:e1004432.

20. Koo T, Lee J, Kim J-S. Measuring and reducing off-target activities of programmable nucleases including CRISPR-Cas9. Mol Cells. 2015;38:475-81.

21. Maeder ML, Gersbach CA. Genome-editing technologies for gene and cell therapy. Mol Ther. 2016;24:430-46.

22. Saito A, Ochiai H, Okada S, Miyata N, Azuma T. Suppression of Lefty expression in induced pluripotent cancer cells. FASEB J. 2013;27:2165-74.

23. Nishimura K, Sano M, Ohtaka M, Furuta B, Umemura Y, Nakajima Y, et al. Development of defective and persistent Sendai virus vector: a unique gene delivery/expression system ideal for cell reprogramming. J Biol Chem. 2011; 286:4760-71.
24. Takahashi K, Tanabe K, Ohnuki M, Narita M, Ichisaka T, Tomoda K, et al. Induction of pluripotent stem cells from adult human fibroblasts by defined factors. Cell. 2007;131:861-72.

25. Zhang YW, Yasui N, Kakazu N, Abe T, Takada K, Imai S, et al. PEBP2aA/CBFA1 mutations in Japanese cleidocranial dysplasia patients. Gene. 2000;244:21-8.

26. Li HL, Gee P, Ishida K, Hotta A. Efficient genomic correction methods in human iPS cells using CRISPR-Cas9 system. Methods. 2015;101:27-35.

27. Hayashi K, Ochiai-Shino H, Shiga T, Onodera S, Saito A, Shibahara T, et al. Transplantation of human-induced pluripotent stem cells carried by selfassembling peptide nanofiber hydrogel improves bone regeneration in rat calvarial bone defects. BDJ Open. 2016;2:15007.

28. Villanueva AR, Mehr LA. Modifications of the Goldner and Gomori one-step trichrome stains for plastic-embedded thin sections of bone. Am J Med Technol. 1977;43:536-8.

29. Harada S, Rodan GA. Control of osteoblast function and regulation of bone mass. Nature. 2003;423:349-55.

30. Zaidi SK, Javed A, Choi JY, van Wijnen AJ, Stein JL, Lian JB, et al. A specific targeting signal directs Runx2/Cbfa1 to subnuclear domains and contributes to transactivation of the osteocalcin gene. J Cell Sci. 2001;114:3093-102.

31. Zaidi SK, Javed A, Pratap J, Schroeder TM, Westendorf JJ, Lian JB, et al. Alterations in intranuclear localization of Runx2 affect biological activity. J Cell Physiol. 2006;209:935-42.

32. Mastushita M, Kitoh H, Subasioglu A, Kurt Colak F, Dundar M, Mishima K, et al. A glutamine repeat variant of the RUNX2 gene causes cleidocranial dysplasia. Mol Syndromol. 2015;6:50-3.

33. Liu T, Gao Y, Sakamoto K, Minamizato T, Furukawa K, Tsukazaki T, et al. BMP2 promotes differentiation of osteoblasts and chondroblasts in Runx2deficient cell lines. J Cell Physiol. 2007;211:728-35.

34. Raya Á, Rodríguez-Pizà I, Guenechea G, Vassena R, Navarro S, Barrero MJ, et al. Disease-corrected haematopoietic progenitors from Fanconi anaemia induced pluripotent stem cells. Nature. 2009;460:53-9.

35. Ma N, Liao B, Zhang H, Wang L, Shan Y, Xue Y, et al. Transcription activatorlike effector nuclease (TALEN)-mediated gene correction in integration-free thalassemia-induced pluripotent stem cells. J Biol Chem. 2013;288:34671-9.

36. Sebastiano V, Maeder ML, Angstman JF, Haddad B, Khayter C, Yeo DT, et al. In situ genetic correction of the sickle cell anemia mutation in human induced pluripotent stem cells using engineered zinc finger nucleases. Stem Cells. 2011;29:1717-26.

37. Wang Y, Zheng C-G, Jiang Y, Zhang J, Chen J, Yao C, et al. Genetic correction of $\beta$-thalassemia patient-specific iPS cells and its use in improving hemoglobin production in irradiated SCID mice. Cell Res Nature. 2012;22:637-48

38. Lian JB, Stein GS, Javed A, Van Wijnen AJ, Stein JL, Montecino M, et al. Networks and hubs for the transcriptional control of osteoblastogenesis. Rev Endocr Metab Disord. 2006;7:1-16.

39. Hojo H, Ohba S, He X, Lai LP, McMahon AP. Sp7/Osterix is restricted to bone-forming vertebrates where it acts as a Dlx co-factor in osteoblast specification. Dev Cell. 2015;37:238-53.

40. Yousfi M, Lasmoles F, Kern B, Marie PJ. TWIST inactivation reduces CBFA1/ RUNX2 expression and DNA binding to the osteocalcin promoter in osteoblasts. Biochem Biophys Res Commun. 2002;297:641-4.

41. Matsubara T, Kida K, Hata K, Ichida F, Meguro H, Aburatani H. BMP2 regulates Osterix through Msx2 and Runx2 during osteoblast differentiation. J Biol Chem. 2008;283:29119-25.

42. Tsuji K, Komori T, Noda M. Aged mice require full transcription factor, Runx2/Cbfa1, gene dosage for cancellous bone regeneration after bone marrow ablation. J Bone Miner Res. 2004;19:1481-9. 